\title{
Hydrate-based Gas Storage Application using Simulated Seawater in the Presence of a Co- promoter: Morphology Investigation
}

\author{
Katipot Inkonga, Viphada Yodpetch ${ }^{\mathrm{a}}$, Hari Prakash Veluswamy ${ }^{\mathrm{b}}$, \\ Santi Kulprathipanja ${ }^{\text {a }}$, Pramoch Rangsunvigit ${ }^{\mathrm{a}, \mathrm{c}}$ * and Praveen Linga ${ }^{\mathrm{d}}$ *
}

${ }^{a}$ The Petroleum and Petrochemical College, Chulalongkorn University, Bangkok 10330, Thailand

${ }^{b}$ Department of Chemical Engineering, Indian Institute of Technology Roorkee, Roorkee 247667, Uttarakhand, India

${ }^{c}$ Center of Excellence in Petrochemical Materials Technology (PETROMAT), Chulalongkorn University, Bangkok 10330, Thailand

${ }^{d}$ Department of Chemical and Biomolecular Engineering, National University of Singapore, Singapore 117575, Singapore

*Corresponding author: $\quad$ Tel: (+66) 2218 4124, Email: Pramoch.r@chula.ac.th Tel: +(65) 6601 1487, Email: Praveen.linga@nus.edu.sg

No of Pages: 4

No of Figures: 6 
(a)

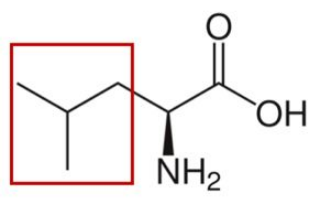

(b)

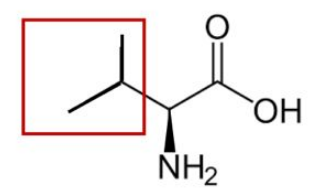

(c)

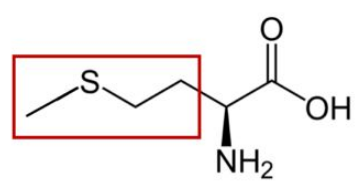

Figure S1 Structure of nonpolar amino acids (a) leucine (b) valine (c) Methionine.

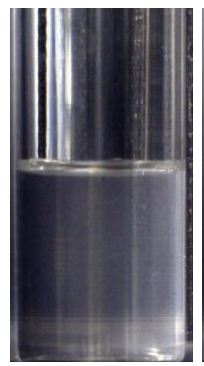

(a) Start Experiment

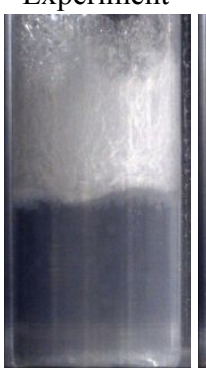

(i) $\mathrm{t}=20 \min (\mathrm{j}) \mathrm{t}=30 \mathrm{~min}$

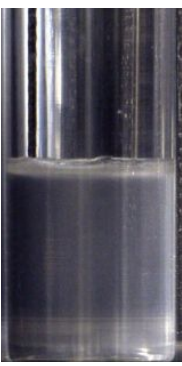

(b) Nucleation

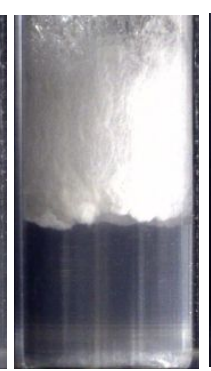

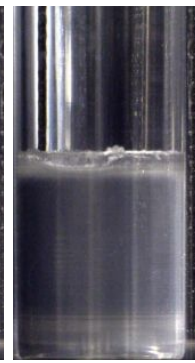

(c) $\mathrm{t}=10 \mathrm{~s}$

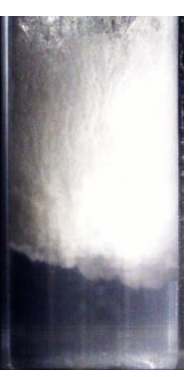

(k) $\mathrm{t}=40 \mathrm{~min}$

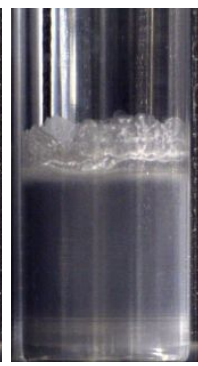

(d) $t=30 \mathrm{~s}$

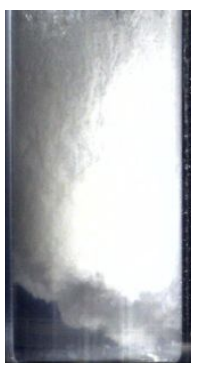

(1) $\mathrm{t}=50 \mathrm{~min}$

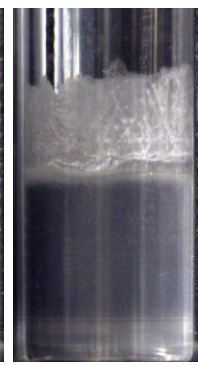

(e) $t=1 \mathrm{~min}$

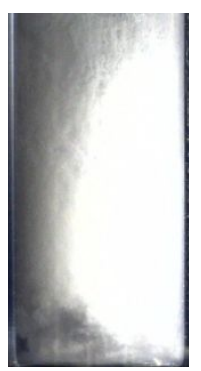

(m) $\mathrm{t}=60 \mathrm{~min}$

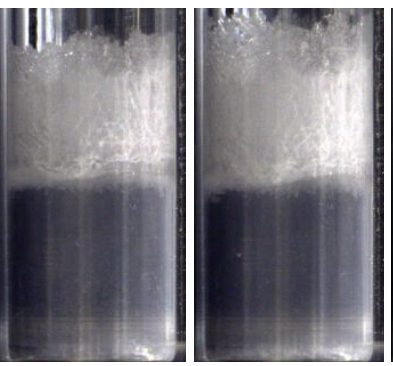

(f) $t=5 \min \quad$ (g) $t=10 \min$

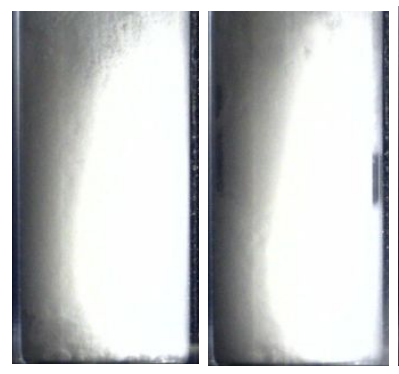

(n) $\mathrm{t}=70 \mathrm{~min}$ h) $t=15 \min$
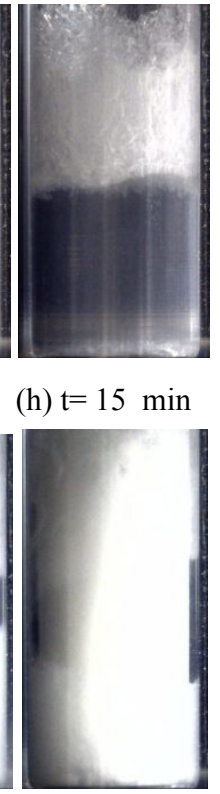

(o) $\mathrm{t}=80 \mathrm{~min}$

(p) $t=90 \min$

Figure S2 Morphology at different time lapses during the mixed methane-THF hydrate formation in the presence of $3.5 \mathrm{wt} \% \mathrm{NaCl}$ at $8.0 \mathrm{MPa}$ and $288.2 \mathrm{~K}$. 

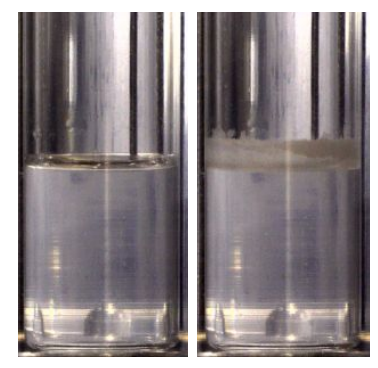

(a) Start

Experiment

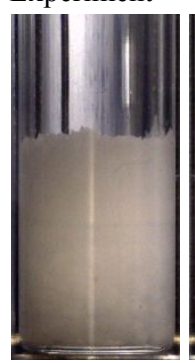

(i) $\mathrm{t}=1 \mathrm{~min}$ $30 \mathrm{~s}$ (b) Nucleation

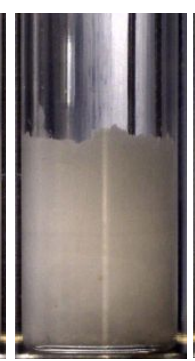

(j) $t=2 \mathrm{~min}$

(k) $\mathrm{t}=2 \mathrm{~min}$ $30 \mathrm{~s}$

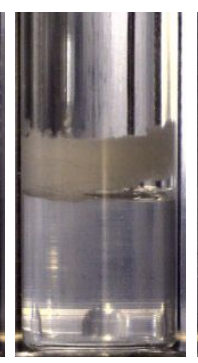

(d) $t=20 \mathrm{~s}$

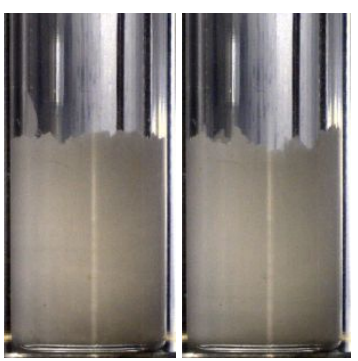

(1) $t=5 \mathrm{~min}$

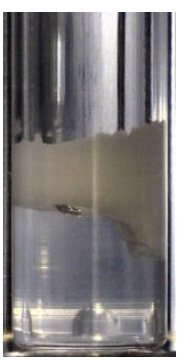

(e) $t=30 \mathrm{~s}$

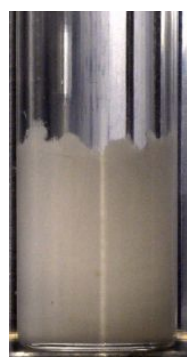

(m) $\mathrm{t}=10 \mathrm{~min}$

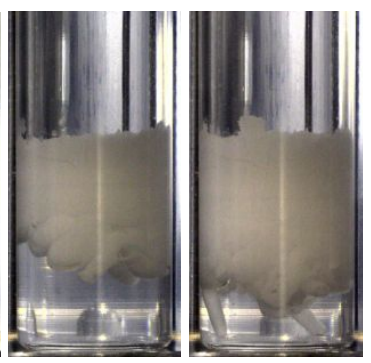

(f) $\mathrm{t}=40 \mathrm{~s}$

(g) $t=50 \mathrm{~s}$
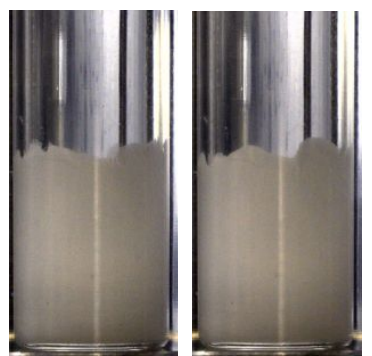

(o) $t=30$ min

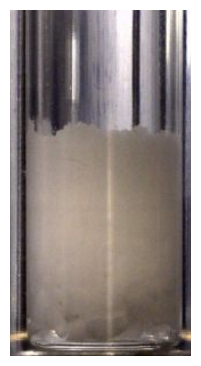

(h) $\mathrm{t}=1 \mathrm{~min}$

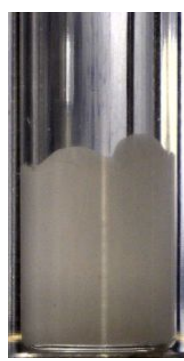

(p) $\mathrm{t}=60 \mathrm{~min}$

Figure S3 Morphology at different time lapses during the mixed methane hydrate formation in the presence of $5.56 \mathrm{~mol} \% \mathrm{THF}$ and $3.5 \mathrm{wt} \% \mathrm{NaCl}$ with $500 \mathrm{ppm}$ SDS at 8.0 MPa and 288.2 K.

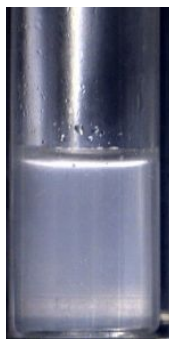

(a) Start Experiment

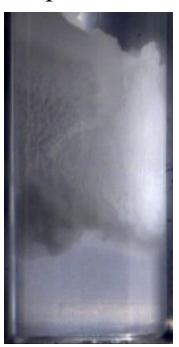

(i) $\mathrm{t}=20 \mathrm{~min}$

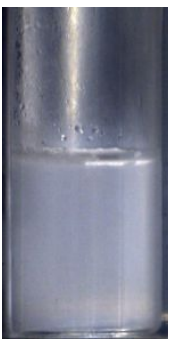

(b) Nucleation

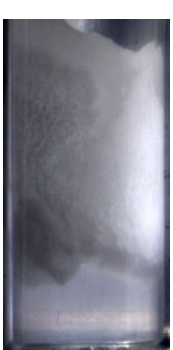

(j) $\mathrm{t}=25 \mathrm{~min}$

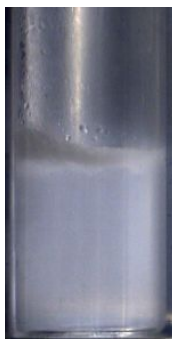

(c) $\mathrm{t}=10 \mathrm{~s}$

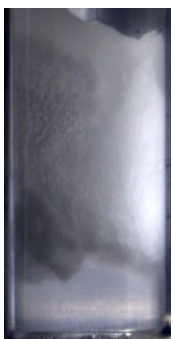

(k) $\mathrm{t}=30 \mathrm{~min}$

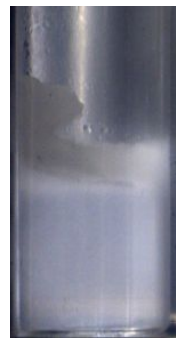

(d) $\mathrm{t}=30 \mathrm{~s}$

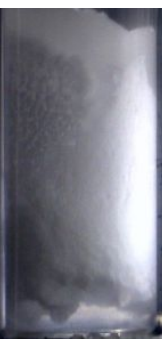

(1) $\mathrm{t}=35 \mathrm{~min}$

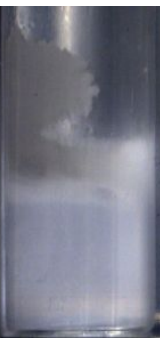

(e) $\mathrm{t}=1 \mathrm{~min}$

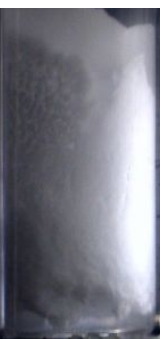

(m) $\mathrm{t}=40 \min (\mathrm{n}) \mathrm{t}=45 \min$

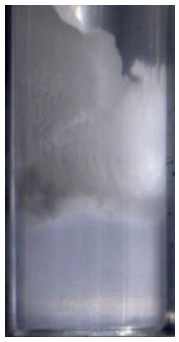

(g) $\mathrm{t}=10 \mathrm{~min}$

(h) $\mathrm{t}=15 \mathrm{~min}$

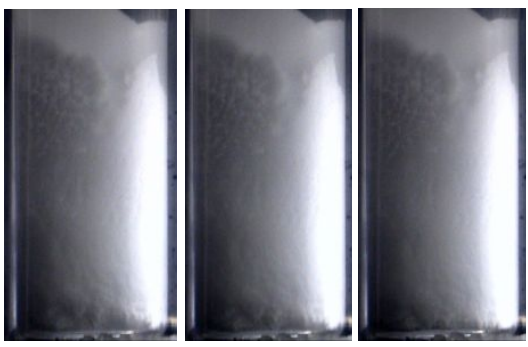

(o) $t=50 \min \quad$ (p) $t=60 \min$ 
Figure S4 Morphology at different time lapses during the mixed methane hydrate formation in the presence of $5.56 \mathrm{~mol} \% \mathrm{THF}$ and $3.5 \mathrm{wt} \% \mathrm{NaCl}$ with $500 \mathrm{ppm}$ Valine at $8.0 \mathrm{MPa}$ and 288.2 K.

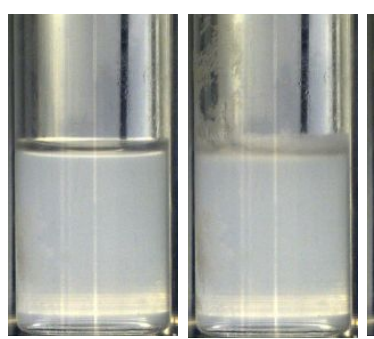

(a) Start Experiment

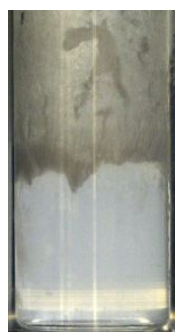

(i) $\mathrm{t}=20 \mathrm{~min}$ (b) Nucleation

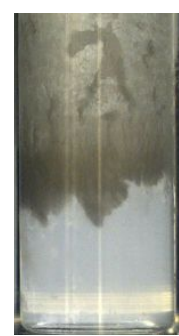

(j) $\mathrm{t}=25 \mathrm{~min}$

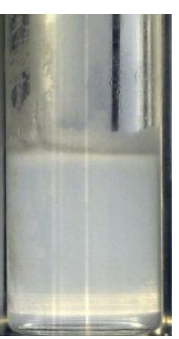

(c) $\mathrm{t}=10 \mathrm{~s}$

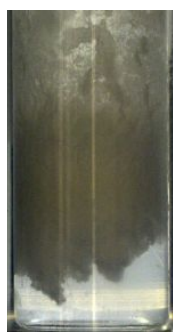

(k) $\mathrm{t}=30 \mathrm{~min}$

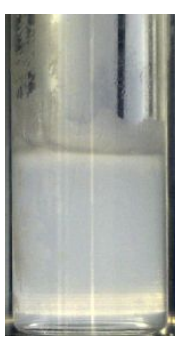

(d) $\mathrm{t}=30 \mathrm{~s}$

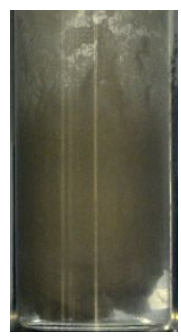

(1) $\mathrm{t}=35 \mathrm{~min}$

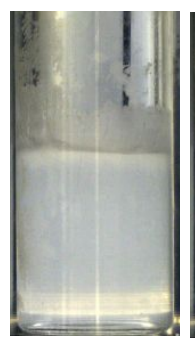

(e) $\mathrm{t}=1 \mathrm{~min}$

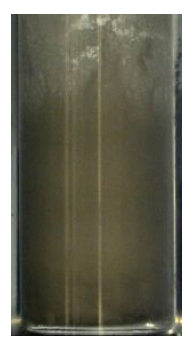

(m) $\mathrm{t}=40 \mathrm{~min}$

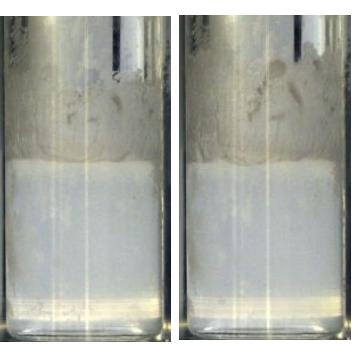

(f) $t=5 \min \quad(g) t=10 \min$

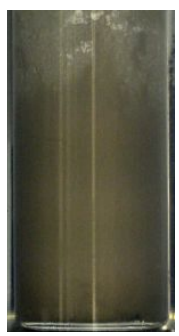

(n) $\mathrm{t}=45 \min \quad(\mathrm{o}) \mathrm{t}=50 \mathrm{~min}$

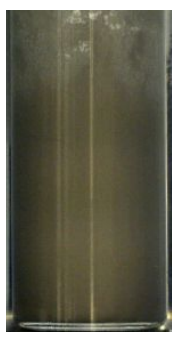

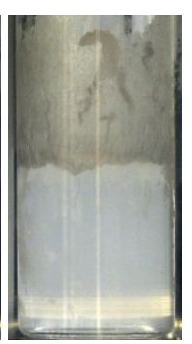

(h) $\mathrm{t}=15 \mathrm{~min}$

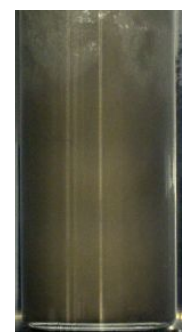

(p) $\mathrm{t}=60 \mathrm{~min}$

Figure S5 Morphology at different time lapses during the mixed methane hydrate formation in the presence of $5.56 \mathrm{~mol} \% \mathrm{THF}$ and $3.5 \mathrm{wt} \% \mathrm{NaCl}$ with $500 \mathrm{ppm}$ Leucine at $8.0 \mathrm{MPa}$ and 288.2 K. 


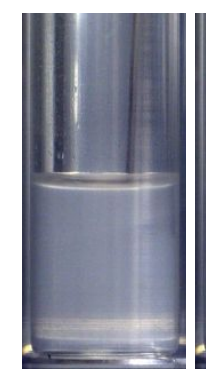

(a) Start

Experiment

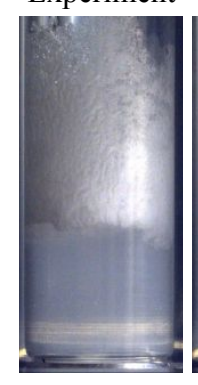

(i) $\mathrm{t}=20 \min \quad$ (j) $\mathrm{t}=25 \mathrm{~min}$

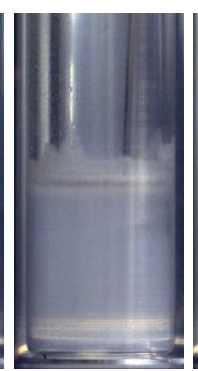

(b) Nucleation (c) $\mathrm{t}=10 \mathrm{~s}$

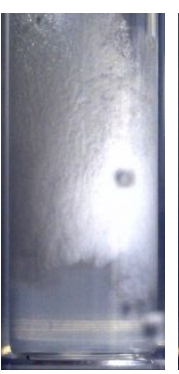

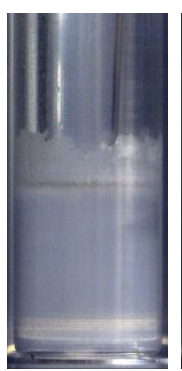

(d) $t=30 \mathrm{~s}$
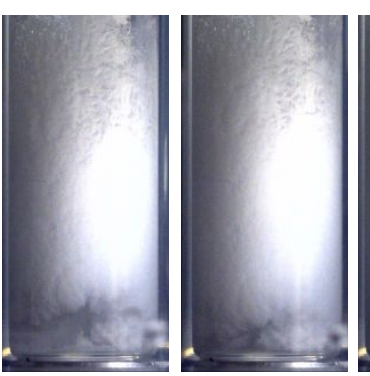

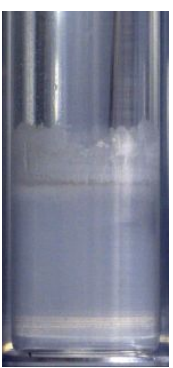

(e) $\mathrm{t}=1 \mathrm{~min}$

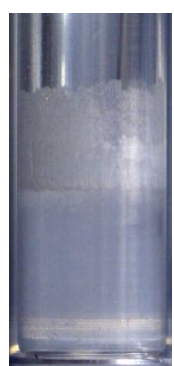

(f) $\mathrm{t}=5 \mathrm{~min}$

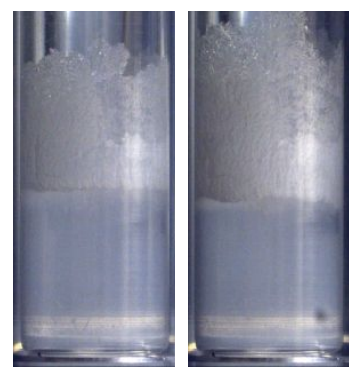

(g) $t=10 \min (h) t=15 \min$
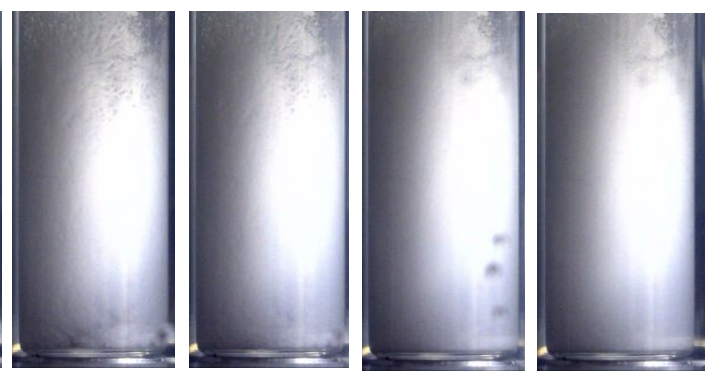

(k) $\mathrm{t}=30 \min (\mathrm{l}) \mathrm{t}=35 \min (\mathrm{m}) \mathrm{t}=40 \min (\mathrm{n}) \mathrm{t}=45 \min (\mathrm{o}) \mathrm{t}=50 \min (\mathrm{p}) \mathrm{t}=60 \min$

Figure S6 Morphology at different time lapses during the mixed methane hydrate formation in the presence of $5.56 \mathrm{~mol} \%$ THF and $3.5 \mathrm{wt} \% \mathrm{NaCl}$ with $500 \mathrm{ppm}$ Methionine at $8.0 \mathrm{MPa}$ and $288.2 \mathrm{~K}$. 